\title{
ON SOME PERTURBATION TECHNIQUES FOR QUASI-LINEAR PARABOLIC EQUATIONS *
}

\author{
Igor Malyshev \\ Department of Mathematics and Computer Science \\ San Jose State University, San Jose, CA 95192
}

\begin{abstract}
We study a nonhomogeneous quasi-linear parabolic equation and introduce a method that allows us to find the solution of a nonlinear boundary value problem in "explicit" form. This task is accomplished by perturbing the original equation with a source function, which is then found as a solution of some nonlinear operator equation.
\end{abstract}

Key words: quasi-linear parabolic equation, heat potentials, boundary value problem, operator equation

AMS subject classification: $\quad 35 \mathrm{~K} 60$

\section{INTRODUCTION}

We need to find $u(x, t)$ from the 1-D quasi-linear parabolic equation in the form:

(1) $\frac{\partial u}{\partial t}-\Delta u+A[u]=f(x, t), \quad x>0, t>0$,

(2) $\mathrm{u}(\mathrm{x}, 0)=0, \mathrm{x}>0$,

(3) $u(0, t)=\gamma(t), t>0,(\gamma(0)=0)$,

where $\mathrm{A}$ is some nonlinear operator (specified in [2] in more detail). Also note

* Received: January 1990, Revised: June 1990 
that a non-zero initial condition case is reducible to (2).

The general quasi-linear parabolic operator $\mathrm{L}$ in the form ([1])

$$
L u=\frac{\partial u}{\partial t}-a_{i, j}\left(x, t, u, u_{x}\right) u_{x_{i} x_{j}}+a\left(x, t, u, u_{x}\right)
$$

requires a rather long list of properties for functions $a_{i, j}$ and $a$ to hold in order to maintain the existence and the uniqueness of the solution of the problem (1)-(3). In our case, $i=j, a_{i, j}=1$ and $a\left(x, t, u, u_{x}\right)=A[u]-f(x, t)$. The complete set of conditions is presented in [2]. Here we simply recollect that a ( $x, t, u, p)$ (p stays for $\left.u_{x}\right)$ is presumed to be continuous in $x, t, u, p$ for $(x, t) \in[0, \infty) \times[0, T],\|u\|$ $\leq \mathrm{M}$ and arbitrary $\mathrm{p}$, and continuously differentiable in $\mathrm{x}, \mathrm{u}, \mathrm{p} ; \gamma(\mathrm{t}) \in \mathrm{C}^{1}[0, \mathrm{~T}]$. We also require that $A[v+w]=A[v]+A[w]+N[v, w]$, where $N$ is some operator linear in $v(w)$ separately, and either $f(x, t)$ or $A[0]$, or $\gamma(t) \neq 0$; otherwise the solution of (1)-(3) is $\equiv 0$, which is not of any interest.

\section{OUTLINE OF THE METHOD}

The method is based on the perturbation techniques introduced in [2] and the heat potentials [3]. Now, we consider a linear initial-boundary value problem induced by (1)-(3):

$$
\begin{aligned}
& \frac{\partial w}{\partial t}-\Delta w=F(x, t), \quad x>0, t>0, \\
& w(x, 0)=0, \quad x>0, \\
& w(0, t)=\gamma(t)
\end{aligned}
$$

with yet unknown source function $F$. Then, we can express $w$ as a sum of a volume potential and a double-layer potential concentrated on $S=\{x=0, t>0\}$ :

$$
w=E * F+E *\left(-\frac{\partial}{\partial x} \mu \delta_{S}\right)
$$




$$
=\int_{0}^{t} d \tau \int_{0}^{\infty} F(\xi, \tau) E(x-\xi, t-\tau) d \xi+\left.\int_{0}^{t} \mu(\tau) \frac{\partial}{\partial \xi} E(x-\xi, t-\tau)\right|_{\xi=0} d \tau .
$$

Due to the "jump formula" for the double-layer potential $\mathrm{W}(\mathrm{x}, \mathrm{t}, \mu)$ (second integral in (4)) as $x \rightarrow 0^{+}$, the unknown density function $\mu(t)$ satisfies the following linear Volterra integral equation of the second kind:

$$
\gamma(t)=\int_{0}^{t} d \tau \int_{0}^{\infty} F(\xi, \tau) E(0-\xi, t-\tau) d \xi+\frac{1}{2} \mu(t)+W(0, t, \mu)
$$

where $E$ is a fundamental solution of the standard linear 1-D heat operator. Since $\mathrm{W}(0, \mathrm{t}, \mu)=0,(5)$ gives $\mu(\mathrm{t})$ explicitly as a linear operator of $\mathrm{F}$, which we denote by $G(F)$ :

$$
\mu(t)=G(F)=2 \gamma(t)+\frac{1}{\sqrt{\pi}} \int_{0}^{t} d \tau \int_{0}^{\infty} F(\xi, \tau) \frac{1}{\sqrt{t-\tau}} e^{-\frac{\xi^{2}}{4(t-\tau)}} d \xi
$$

Then, representing the solution of (1)-(3) in the form $u=w+v$, we find that the function $v(x, t)$ has to satisfy the following equation (with zero initial and boundary data):

$$
\frac{\partial v}{\partial t}-\Delta v+A[v]+N[v, w]=f(x, t)-F(x, t)-A[w]
$$

where $\mathrm{w}$ is defined by (4), (6) and $\mathrm{N}$ is a linear operator with respect to $\mathrm{v}$.

Since $F$ is not yet known, we can try to find it so that right side of (7) becomes identically equal to 0 . After this is accomplished, we obtain a homogeneous problem for $v$, which together with the uniqueness theorem [1] implies that $v \equiv 0$. As a result, $u=w$, and the solution of the nonlinear problem (1)-(3) is found in explicit form (4). Since $\mathrm{W}(\mathrm{x}, \mathrm{t}, \mathrm{G}(\mathrm{F}))$ is a convolution in the time variable only 


$$
\begin{aligned}
& W(x, t, G(F))=E(x, t) * G(F))(t) \delta_{S}=\left.\int_{0}^{t} G(F)(\tau) \frac{\partial}{\partial \xi} E(x-\xi, t-\tau)\right|_{\xi=0} d \tau \\
& =\frac{x}{4 \sqrt{\pi}} \int_{0}^{t} G(F)(\tau) \frac{1}{(t-\tau)^{3 / 2}} e^{-\frac{x^{2}}{4(t-\tau)}} d \tau,
\end{aligned}
$$

the corresponding equation for $\mathrm{F}$ in the form

$$
F(x, t)=f(x, t)-A\left[E * F+E * G(F) \delta_{S}\right]
$$

is an operator equation, where $\mathrm{A}$ is some nonlinear operator applied to the linear Volterra integral operator from (4). If (8), being expressed in the form $B[F]=F$, has a unique fixed point and, for example, Picard's method is applied, then $F_{n} \rightarrow F$, $f-F_{n}-A\left[E * F_{n}\right] \rightarrow 0, \quad v_{n} \rightarrow 0, \quad w_{n}=u_{n}=E * F_{n} \rightarrow E * F=u$.

As in [2], stability analysis is based on convergence of the Picard method, representation (4), and the fact that $\mathrm{w}$ in (4) continuously depends on $\mathrm{F}$ in the following sense: if $\left\|F-F^{\prime}\right\| \leq \varepsilon$, then $\left\|w-w^{\prime}\right\| \leq T \varepsilon$, where $T$ defines the thickness of the layer. Of course, the spaces and the norms will have to be chosen depending on the method of solution we apply to (8), specific operator A, etc. In general, the accuracy of our approximation of the solution $u$ can be as good as we wish, allowing a sufficient number of steps in the process of solving (8) for F.

It is also worth mentioning that the class of functions $\mathrm{F}$ may be quite arbitrary, since $F$ is not given as input data and depends entirely on our choice and ability to solve the corresponding equation. For different choices of $F$ we should expect different types of solutions of (1) ranging from weak to classical.

\section{EXAMPLE}

Let $A[u]=u^{2}(x, t)$, that is $a(x, t, u, p)=u^{2}-f(x, t)$ in (1), and $N$ in (7) has 
the form: $N[v, w]=2 \mathrm{v} w$. Now, we consider (8) with operator $B$ in the form:

(9) $\quad F=B[F]=f(x, t)$

$-\left(\int_{0}^{t} d \tau \int_{R^{3}} F(\xi, \tau) E(x-\xi, t-\tau) d \xi+\left.\int_{0}^{t} G(F)(\tau) \frac{\partial}{\partial \xi} E(x-\xi, t-\tau)\right|_{\xi=0} d \tau\right)^{2}$.

Solving (9) means finding a fixed point of the operator B.

Let $\mathrm{f} \in \mathrm{C}([0, \infty) \times[0, \mathrm{~T}])$ - the Banach space of continuous functions with standard uniform norm. We seek solution of (9) in a closed ball $U_{F}=\{F:\|F\| \leq$ $M\}$ in the Banach space $C$, centered at the origin and with radius $M$ specified later. It can be proved that $B$ is a contraction on $U_{F}$ for sufficiently small $T$, since

$$
\begin{aligned}
& \left\|\mathrm{E} * \mathrm{~F}_{1}-\mathrm{E} * \mathrm{~F}_{2}\right\| \leq\left\|\mathrm{F}_{1}-\mathrm{F}_{2}\right\| \mathrm{T}, \\
& \left\|\mathrm{E} * \mathrm{~F}_{1}+\mathrm{E} * \mathrm{~F}_{2}\right\| \leq 2 \mathrm{M}_{\mathrm{F}} \mathrm{T}, \\
& \left\|\mathrm{G}\left(\mathrm{F}_{1}\right)-\mathrm{G}\left(\mathrm{F}_{2}\right)\right\| \leq 2\left\|\mathrm{~F}_{1}-\mathrm{F}_{2}\right\| \mathrm{T}, \\
& \left\|\mathrm{E} *\left(\mathrm{G}\left(\mathrm{F}_{1}\right)-\mathrm{G}\left(\mathrm{F}_{2}\right)\right) \delta_{\mathrm{S}}\right\| \leq\left\|\mathrm{F}_{1}-\mathrm{F}_{2}\right\| \mathrm{T}, \\
& \left\|\mathrm{E} *\left(\mathrm{G}\left(\mathrm{F}_{1}\right)+\mathrm{G}\left(\mathrm{F}_{2}\right)\right) \delta_{S}\right\| \leq 2 \mathrm{M}_{\mathrm{F}} \mathrm{T}, \\
& \left|\mathrm{B}\left[\mathrm{F}_{1}\right]-\mathrm{B}\left[\mathrm{F}_{2}\right]\right|=\left|\mathrm{E} *\left(\mathrm{~F}_{1}-\mathrm{F}_{2}\right)+\mathrm{E} *\left(\mathrm{G}\left(\mathrm{F}_{1}\right)-\mathrm{G}\left(\mathrm{F}_{2}\right)\right) \delta_{\mathrm{S}}\right| \\
& \times\left|\mathrm{E} *\left(\mathrm{~F}_{1}+\mathrm{F}_{2}\right)+\mathrm{E} *\left(\mathrm{G}\left(\mathrm{F}_{1}\right)+\mathrm{G}\left(\mathrm{F}_{2}\right)\right) \delta_{S}\right| \\
& \leq 8 \mathrm{M} \mathrm{T}\left\|\mathrm{F}_{1}-\mathrm{F}_{2}\right\|,
\end{aligned}
$$

and, finally, 
(10)

$$
\left\|\mathrm{B}\left[\mathrm{F}_{1}\right]-\mathrm{B}\left[\mathrm{F}_{2}\right]\right\| \leq \rho_{0}\left\|\mathrm{~F}_{1}-\mathrm{F}_{2}\right\| \text {, where } \rho_{0}=8 \mathrm{MT}^{2}<1
$$

In addition, operator B satisfies the following condition at the center (see [5], "contraction on a ball theorem"):

(11) $\|0-\mathrm{B}[0]\|=\|\mathrm{f}\|<\left(1-\rho_{0}\right) \mathrm{M}$, which, together with (10), implies that $U_{F}$ remains invariant under the operator $B$, and the method of successive approximations is convergent. The necessity to satisfy (10) and (11) simultaneously, gives the basis for the proper choice of $M$ and T. The complete analysis is quite simple and thus we shall supply one example only:

$$
\mathrm{M}=3\|\mathrm{f}\|, \mathrm{T}^{2}=1 /(48\|\mathrm{f}\|) \text {, and } \rho_{0}=8 \mathrm{M} \mathrm{T}^{2}=1 / 2 \text {. }
$$

Obviously, both (10) and (11) are satisfied.

Hence, the unique solution of (8) can be first found by the Picard method in some sufficiently thin layer $[0, \infty) \times\left[0, \mathrm{~T}_{1}\right]$.

To extend this process onto the next $\left[\mathrm{T}_{1}, \mathrm{~T}_{2}\right]$ - layer we solve (8) in the class of functions that coincide with the just found $F(x, t)$ in the layer from the previous step. This, and the fact that $M$ is an absolute constant, allow us to obtain the next estimate for $t \in\left[T_{2}, T_{3}\right]$ identical to (10), where $T_{2}-T_{1}$ substitutes $T_{1}$. That is, the extension onto arbitrary $[0, T]$ - layer can be accomplished in a finite number of equal "steps".

The method is stable in C-metric both at the stage of finding $\mathrm{F}$ and later, when solution is calculated in the form (4), (6).

\section{Remarks.}

1. This method may be applicable to a wide range of cases with different linear/nonlinear operators A (both for Cauchy and boundary value problems) that comply with the assumptions mentioned in Sec.1. It should be noted that those conditions are not related to equation (8) (the equation (8) itself is of a separate interest and can be considered under a variety of assumptions on function $F$ and operator $A$ ). We need them exclusively to ensure that the only solution possible for the 
homogeneous equation (7) is $v \equiv 0$. Any other set of conditions on coefficients $a_{i, j}$, a , and boundary data $\gamma$ will do as long as uniqueness in (7) can be maintained.

2. The method can be used separately as a constructive tool for proving existence and uniqueness theorems in a variety of cases.

3. The method is not restricted to quasi-linear equations. It can be tried on some other nonlinear and linear (including variable coefficients) cases.

4. For the case of $A[u]=u u_{x}$, as in [2], the unique solvability of the corresponding equation (8) can be proved, thus giving the solution of (1)-(3) in the form (4).

\section{REFERENCES}

[1] Ladyzenskaja, O. N., et al., Linear and Quasi-linear Equations of Parabolic Type, AMS, Providence, (1968).

[2] Malyshev, I.G., On perturbation techniques in a model case from planetary waves theory, to appear in: "Nonlinear Structures in Physical Systems - Pattern Formation, Chaos and Waves," Springer-Verlag , (1990).

[3] Vladimirov, V.S., Equations of Mathematical Physics, Marcel Dekker, (1971).

[4] Gradshteyn, I.S., Ryzhik, I.M., Table of Integrals, Series and Products, Academic Press, (1980).

[5] Kreyszig, E., Introductory Functional Analysis with Applications, J. Wiley \& Sons, (1978). 


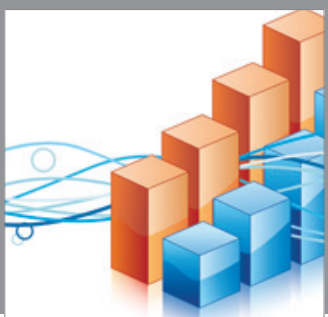

Advances in

Operations Research

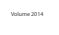

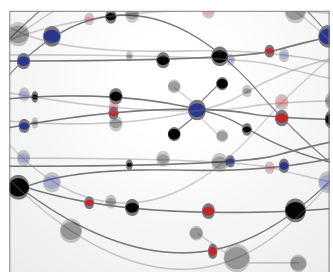

\section{The Scientific} World Journal
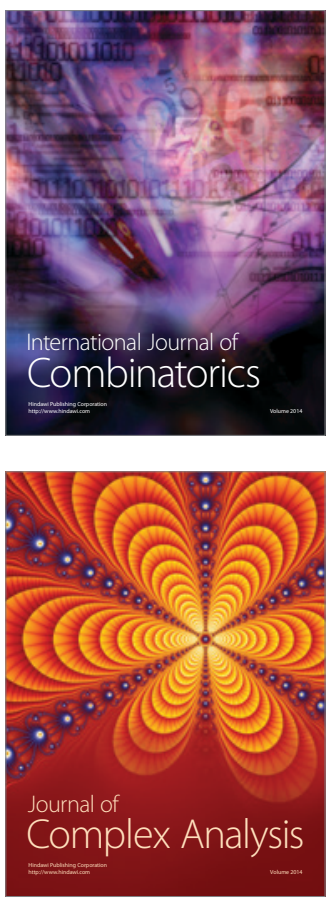

International Journal of

Mathematics and

Mathematical

Sciences
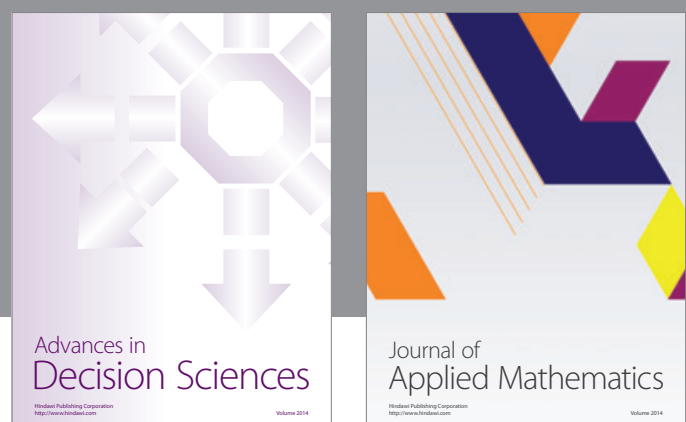

Journal of

Applied Mathematics
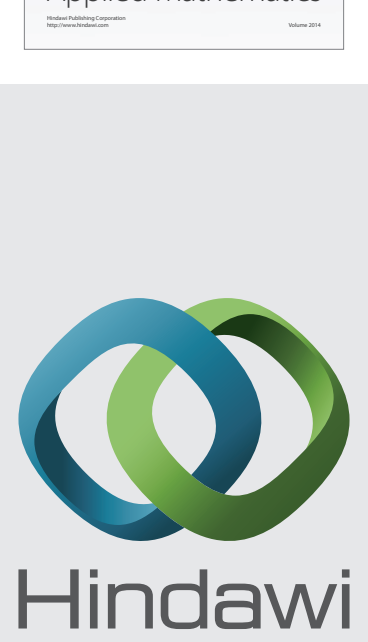

Submit your manuscripts at http://www.hindawi.com
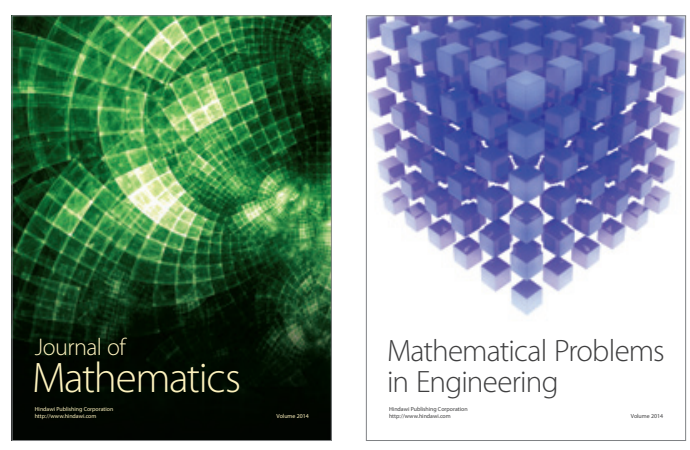

Mathematical Problems in Engineering
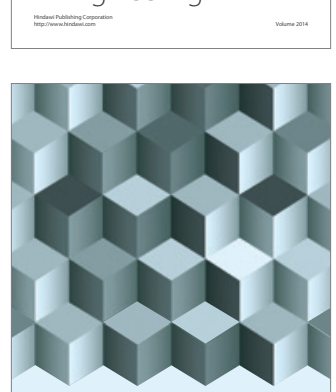

Journal of

Function Spaces
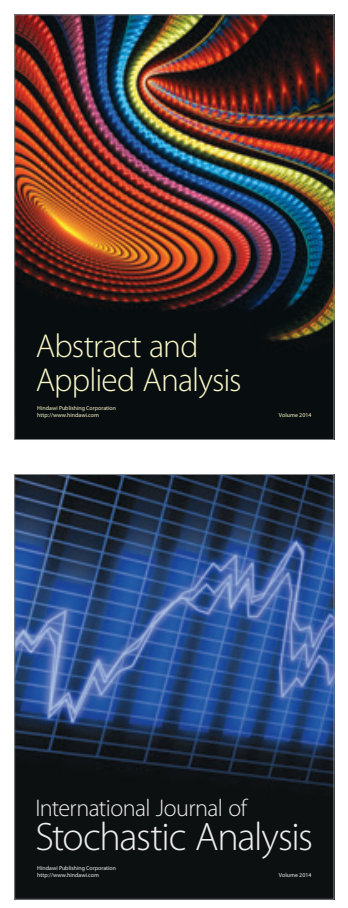

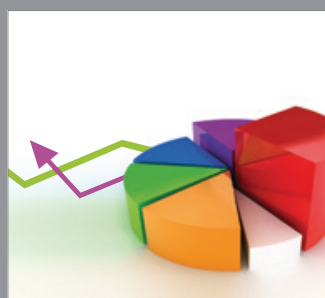

ournal of

Probability and Statistics

Promensencen
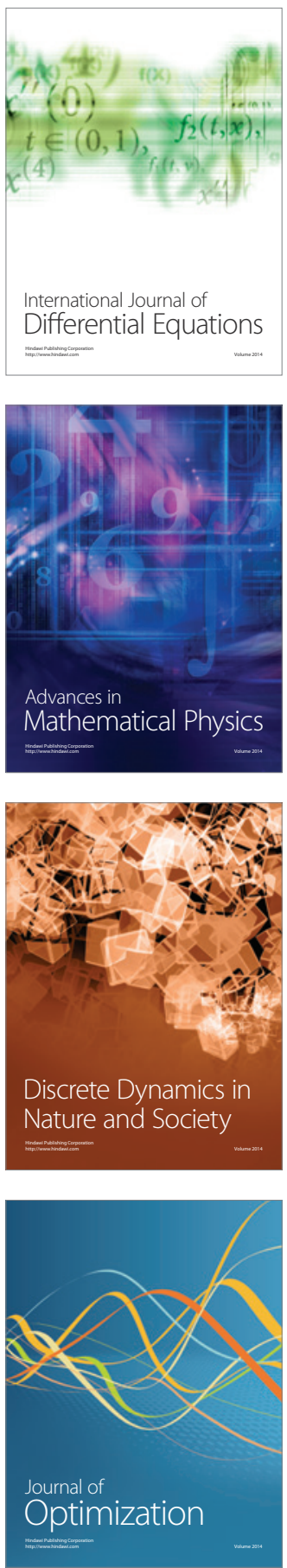